\title{
Personal Fable: Optimistic Bias in Cigarette Smokers
}

\author{
Marianna Masiero $^{1,2,}{ }^{*}$ Claudio Lucchiari ${ }^{1,2}$; Gabriella Pravettoni $^{1,2}$ \\ ${ }^{1}$ Department of Health Sciences, University of Milan, Milan, Italy \\ ${ }^{2}$ Applied Research Unit for Cognitive and Psychological Science, European Institute of Oncology, Milan, Italy \\ ${ }^{*}$ Corresponding author: Marianna Masiero, Department of Health Sciences, University of Milan, P. O. Box: 20123, Milan, Italy. Tel: +39-0250321228, Fax: +39-0250321240, E-mail: Mari- \\ anna.masiero@guest.unimi.it
}

Received: June 3, 2014; Revised: September 8, 2014; Accepted: September 15, 2014

\begin{abstract}
Background: Several empirical studies have shown the attitude of smokers to formulate judgments based on distortion in the risk perception. This alteration is produced by the activation of the optimistic bias characterized by a set of the unrealistic beliefs compared to the outcomes of their behavior. This bias exposes individuals to adopt lifestyles potentially dangerous for their health, underestimate the risks and overestimate the immediate positive effects.

Objectives: This study aimed to analyze the relationship between optimistic bias and smoking habits. In particular, it was hypothesized that smokers develop optimistic illusions, able to facilitate the adoption and the maintenance over time of the unhealthy lifestyles, such as cigarette smoking, and the former smokers could acquire a belief system centered on own responsibility.

Patients and Methods: The samples ( $\mathrm{n}=633$, female $=345$, male $=288)$ composed of smokers (35.7\%), ex-smokers (32.2\%) and nonsmokers (32.1\%). Each participant filled out two questionnaires including The Fagerström test and the motivational questionnaire as well as a set of items measured on a Likert scales to evaluate health beliefs.

Results: The results confirmed the presence of the optimistic bias in comparative judgments, and the attitude to overestimate the effectiveness of their preventive behaviors in the smokers.

Conclusions: Cognitive bias in risk perception may influence health behaviors in negative way and reinforce cigarette smoking over the time. Future research should be conducted to identify the better strategies to overtake this cognitive bias to improve the quitting rate.
\end{abstract}

Keywords:Decision Making; Nicotine Dependence; Bias; Risk

\section{Background}

For a long time, the study of the addictive behavior has been based on a biological model, which highlighted the role of brain chemistry and neurological considerations. In this approach, nicotine dependence has been explained as a consequence of limbic and cortical structures malfunctioning, that was produced by a dopaminergic up-regulation. Actually, nicotine alters the reinforcement signal processing in ventral regions of the basal ganglia thus determining a brain neuro-adaptation to the substance. However, starting by the 1990s a new cognitive model introduced further perspective on smoking. These models are essentially based on the study of risk, since risk dis-perception was considered a major factor in favoring initiation and maintaining an unsafe behavior over the time $(1,2)$.

While previous psychological research had stressed the role of the motivational factors and impulsiveness (3), the new paradigm suggested that smokers' mental models and their belief system might also be considered to understand individual behaviors and decision making, in particular when risks should be weighted on benefits to follow the best option (e.g. smoking versus nonsmoking) (4).
According to Slovic $(5,6)$ an individual may take disadvantageous decisions not only when affective components are purposely manipulated (e.g. in marketing actions), but also when cognitive distortions and biased beliefs are present. Actually, research on smokers' behaviors has shown that they tend to underestimate both the long-term and short-term risks of the tobacco consumption. Consequently, smokers' judgment tends to be driven by anticipatory feelings elicited by previous experiences and consolidated beliefs (7).

Generally speaking, we may state that risk perception is the result of an intrasubjective cognitive assessment. The information processing involves both cognitive and emotional processes. For this reason the risk perception cannot be considered a pure logic and objective evaluation. In particular, three different elements should be taken into consideration to understand health-related risk assessment including the perceived vulnerability, the preventive efficacy and unwarranted optimism. The perceived vulnerability is the degree to which an individual feels to be personally exposed to health consequences due to their own behavior. A high level of vulnerability in smokers is generally associated with a: 
- high level of motivation to give up smoking (8);

- high probability to be involved in smoking cessation programs;

- high likelihood to do a real attempt to give up (9)

It has also been observed that cancer patients with high level of perceived vulnerability generally show a higher motivation to quit (10), and a better success rate (11). The preventive efficacy is related to the belief of an individual to be able to carry out preventive actions (e.g. physical activity) to obtain health benefits. Individuals with high levels of preventive efficacy believe that their decisions (e.g. to stop smoking) will actually preserve their future health. Smokers motivated to give up generally show high level of preventive efficacy and perceive higher benefits associated with smoking discontinuation; However, the former smokers who report to perceive high level of health benefits often fail to sustain their abstinence over time $(9,12)$.

Finally, the unwarranted optimism, also called optimistic bias (13) is a cognitive bias that push people to believe to be invulnerable to a potential risk's source $(14,15)$, allowing them to feel a sense of control over the effects of their choices. This bias leads people to underestimate risks when considering themselves, while being more realistic (or pessimistic) when considering other people's behaviors. The optimist bias entails a "first-person evaluation" as opposed to a "third-person evaluation"; for instance, smokers are usually more optimistic about themselves rather than others. Thus, when they use a first-person evaluation of smoking-related risks, they are much more optimistic than when they evaluate others' risks (e.g. "I can stop when I want" or "Since my grandfather died 80 years old smoking 20 cigarettes a day, I've got good genes"). This optimistic illusion seems to be the result of two distinct mental processes (16-19):

-The illusion of control, due to the overestimation of preventive behavior efficacy (e.g. increased physical activity that decreases the perceived need to stop). The illusion of control leads smokers to the false belief that they will able to stop when they want. In this way, they categorize smoking as a controllable and removable behavior. Only later, after repeated failed quit attempts, they come to understand to be nicotine-dependent. For instance, among occasional smokers, namely who smoke less than a cigarette a day, only $15 \%$ think that within 5 years could become a heavy smoker, thus developing a chronic dependence to nicotine. Moreover, among heavy smokers, 32\% consider that within 5 years they will still be a smoker, while $68 \%$ believe that will interrupt. In reality, the official statistics show that after 5 years $70 \%$ of people keep on smoking (20).

- The need to preserve a good self-esteem level. Indeed, one's self-esteem is generally threatened when a risk is not avoided. At the opposite, one's self-esteem increases when self-efficacy is high $(21,22)$.

In this way, smokers develop illusory beliefs to justify their behaviors and reduce the negative feelings associ- ated to the adoption of a risk choice that could be avoided. Being able to find arguments in favor of others' risks (e.g. a high genetic vulnerability), smokers succeed in managing the cognitive dissonance due to the mismatch between risk perception ("smoking may be hazardous") and the actual behavior (14). This optimistic thinking is linked to the memory functioning, since a recurrent behavior is more easily accessible, and due to a habitation effect, they are considered acceptable though being risky. This implies that, when a person assesses specific levels of risk (e.g. the likelihood to develop a lung cancer due to heavy smoking) available memories will guide judgment instead of an objective assessment $(23,24)$. Finally, the optimistic bias may be linked to so-called wishful thinking including cognitive distortions produced by desirable situation, events, subject and/or object evaluation. This distortion leads people to consider the occurrence of an event more likely than another only because it is more desirable. For instance, smokers may conclude that smoking is not riskier than other behaviors, just because this is a wishful consideration.

It is important to note that both adolescent and adult smokers are generally able to recognize the hazardous smoking in a long-run perspective and the existence of smoking-related diseases (e.g. lung cancer, cardiovascular problem and other cancer syndrome) $(4,25)$.They show to have an adequate information about these issues; nevertheless, they show ambiguous attitudes towards risk, since they don't relate their choices to health and wellbeing.

\section{Objectives}

The present study aimed to analyze the influence of the optimism bias and illusionary beliefs that support smoking initiation, consolidation and maintenance over time. Moreover, this study aimed to evaluate four main hypotheses as follows:

1- Since smoking-related issues are common in the whole society, in smokers and nonsmokers, we hypothesized that smokers with respect to nonsmokers show an optimistic bias, being more benevolent when evaluating first-person risks than third-person ones. At the opposite nonsmokers and former smokers should be more realistic, showing balanced judgments.

2- We hypothesized that former smokers develop a specific belief system to support their abstinence. In particular, we supposed former smokers having higher smoking-related risk perception than smokers. At the same time, we argued that former smokers feel to modulate the effects of previous smoking on their health by their actual behavior.

3- Finally, since risk perception and health-related beliefs are modulate by behavioral, psychological and demographic variables, we hypothesized that age, gender, smoking habits (nicotine dependence) and motivation to quit should affect the smokers' cognitive distortions. 


\section{Patients and Methods}

\subsection{Subjects}

The sample was recruited in collaboration with the Interdisciplinary Research Center on Decision (IRIDe) of the University of Milan. The research was conducted from January 2013 until June 2013. The sample is made by 633 volunteer participants (Female $=54.5 \%$; Male $=45.5 \%$ ), recruited through internet sites. During a telephone contact, all the needed information was delivered and if the subject agreed to participate in the study, an e-mail with the study description, the informant consent and a link to an online questionnaire was sent to him/her. The time to complete the protocol was about 20 minutes for all conditions. Subjects had the possibility to contact a researcher by an e-mail or telephone on demand. After the completion of the procedure, each subject was contacted for a fast debrief. An opportunistic sampling method was used. The mean age of the samples was 48.01 years (standard deviation $=15.203$ ), with an age range of 19-74 years. The samples were classified into three clusters: smokers (35.7\%), ex-smokers (32.2\%), and nonsmokers (32.1\%).

\subsection{Instruments}

Two standardized questionnaires were used:

-The Fagerstrom test for nicotine dependence (FTND) (26) to assess the dependence level both physiological and psychologically;

- A motivational questionnaire to evaluate the intention to give up smoking (27);

To evaluate smoking-related beliefs, we used a set of Likert scale questions. The Fagerström test for nicotine dependence is a 6-item self-administered questionnaire. The scale evaluates three main dimensions including the average daily amount of cigarette smoked, the nicotine compulsion, and the general level of dependence. The total score ranges from a minimum of the 0 points to a maximum of the 10 points, with the following meanings: 0-2 mild dependence, 3-4 not severe dependence, 5-6 strong dependence, 7-10 very strong dependence.

The motivation to give up smoking (27) consists of four multiple-choice questions; to each is assigned a score ranging from 1 to 4 . The total score allow to classify smokers into one of four motivational clusters: 4-6 low (not yet seriously considered to give up smoking); 7-10 middle (the person evaluated both the benefits of quitting and the risks of smoking); 11-14 high (there are moments in which the person is determined to quit smoking); 15-19 very high (the person is ready to give up smoking).

To assess the system of subjects' beliefs, we collected a set of 11 assertions (Appendix 1) adapted by previous works $(12,14)$. The translation and the cultural adaptation of items were performed by a panel of expert, including two psychologists, one counselor expert in tobacco cessation and one professional English to Italian translator. To study the face validity of items, we asked 20 subjects (all smokers) to indicate whether the questions were clear, understandable, and in a logical order. To further validate our version of the instrument, we collected data from 20 students of the University of Milan $(20$ smokers, 20 former smokers, and 20 nonsmokers) in a pilot phase of the study. We performed a test-retest study, asking participants to answer to the same items three months after the first trial, finding a mean test-retest correlation (Spearman's Rho) of 0.88 (range, 0.82-0.96).

Subjects were asked to rate themselves according to each assertion on a 4-point Likert scale. Each item has been built specifically to describe beliefs and opinions with respect to smoking-related risks and dependence issues. Consequently, items do not assess the individual's knowledge levels, since we wanted to assess biased cognitive processes rather than notions. The first 7 items contain assertions on risk perception. These items are semantically constructed to assess the self-oriented (firstperson perspective) risk perception in contrast to a general risk perception (third-person perspective). Items 8 to 11 consider smoking-related myths and cognitive strategies used to cope with tobacco-related risks.

\section{Results}

Data were processed using the (SPSS, IBM, USA) version 20.0. Descriptive statistics were used to analyze sample characteristics. Most smokers (63\%) had a moderate to high nicotine-dependence level as measured by the Fagerström test. At the same time, the $41.7 \%$ of smokers reported a high motivation score, and most fall between high and middle level. Although our sample is quite heterogeneous, most participants had a strong addiction ( mean of daily cigarettes $=20.181$, standard deviation $=12.246$ ) and good motivation to quit. A series of cross tables were created to find associations between the answers and smoking clusters (smokers, ex-smokers and nonsmokers). The chi-square test was used to evaluate statistical differences.

Statistically significant effects were found in items 2, 3, 4,8 , and 11 showing different distributions for the three clusters. In particular, answering to item 2 many smokers reported to doubt that the cigarette smoking could be a possible cause of death, while former and nonsmokers reported more realistic judgments $\left(\mathrm{X}^{2}=25.469, \mathrm{df}=6, \mathrm{P}=\right.$ 0.000 ) with respect to well-known statistics. This result is particularly important because when the subjective perspective (first-person risk evaluation) is substituted by a general perspective (risk for others), the optimistic bias disappeared (Figure 1).

Also, answers to item $3\left(\mathrm{X}^{2}=28.240, \mathrm{df}=6 \mathrm{P}=0.000\right)$ and $4\left(X^{2}=23.436, d f=6, P=0.001\right)$ showed different distributions. These items refer to the smokers' confidence in controlling their behavior. Consequently, we can say that smokers tend to underestimate the power and the salience of the nicotine addiction. In the third area, we analyzed the preventive strategies enacted by respondents to contrast the side-effects of tobacco consump- 
tion. Different distributions were observed in item $8\left(\mathrm{X}^{2}\right.$ $=23.545, \mathrm{df}=6, \mathrm{P}=0.001)$ and item $11\left(\mathrm{X}^{2}=13.724, \mathrm{df}=6\right.$, $\mathrm{P}=0.033)$. Smokers compared to nonsmokers trust more on the power of the physical activity to contrasts the cigarette smoking negative effects. Furthermore, smokers and ex-smokers seem to underestimate the association between tobacco consumption and lung cancer (Figure 1). Smokers probably tend to develop this illusory belief to contrast the mismatch between the pleasure for the smoking (hedonistic dimension) and the health consequences. Coherently with our second hypothesis, former smokers probably need to believe that their previous behavior won't have severe consequences on their future health; otherwise, remaining abstinence could be perceived as useless.

To address our third hypothesis, a similar analysis was carried out on smokers considering motivational to give up groups (low, middle, high) as measured by the motivational questionnaire. However, no significant difference was found in judgments and beliefs among these groups. The role of gender and age was also evaluated. A statistical difference was found at the item 7: the male ex-smokers reported more pessimistic evaluations on the association between cigarette smoking and lung cancer than female smokers $\left(\mathrm{X}^{2}=13.553, \mathrm{df}=3, \mathrm{P}=0.004\right)$.

Four categories were considered to examine the effect of age: $19-40$ years; $41-50$ years; $51-60$ years and 61-75 years. Answers to the item 2, 4, 6 and 10 showed different distributions for age categories. More in details, at the item $2\left(X^{2}=20.380, \mathrm{df}=9, \mathrm{P}=0.016\right)$ smokers under 40 and smokers over 50 tend to underestimate the smoking-related risks. At the opposite, nonsmokers between 19 and 40 years showed higher awareness $\left(X^{2}=17.402 \mathrm{df}=9, \mathrm{P}=\right.$ 0.043 ) about smoking-related risks.

At item 4, younger smokers (aged between 19 and 40) reported to underestimate the strength physical depen- dence more than older smokers $\left(X^{2}=20.833, \mathrm{df}=9, \mathrm{P}=\right.$ 0.013 ). Indeed, they believed that cigarette smoking was an easily controllable behavior. In the clusters of 51-60 and 61-75 years, an inversion of this trend was observed. The younger ex-smokers (19-40 years) tended to underline their chances of contrasting disease development due to protective behaviors $\left(X^{2}=30.771, \mathrm{df}=9, \mathrm{P}=0.000\right.$ ). This trend was in accordance to answers to item 10 , since smokers over 60 underestimated the risk to develop the lung cancer, if they had smoke just for few years $\left(\mathrm{X}_{2}=\right.$ 29.352, $\mathrm{df}=9, \mathrm{P}=0.001$ ). Otherwise, smokers under 60 admitted this risk. It is interesting to note that the nicotinedependence level increased during time $\left(X^{2}=39.628\right.$, df $=9, P=0.000$ ), since smokers in clusters 51-60 and 61-75 years reported higher level of dependence as measure by the Fageström test. This datum suggests that smokers with high levels of nicotine-dependence (and then the number of cigarettes consumed) also reported a heavily biased smoking-related risk perception.

\section{Discussion}

In the 2011, the World Health Organization report on the global tobacco epidemic as stressed that the cigarette smoking is a first cause of death in the world. Each year, tobacco consumption kills six millions of people. Many of these people develop a respiratory or cardiovascular disease due to smoking; however, few of these individuals seem to be aware of this great problem. In 2010, it was conducted a study in several American hospitals, and it was discovered that the $47.6 \%$ of smokers admitted at the emergency department for acute respiratory care did not believe that the real cause was cigarettes smoking (28). In smokers is strong the tendency to underestimate all smoking-related risks. The need to defend the self-esteem induces the addicted subject to develop a series of illusion and false beliefs to support the choice to keep on smoking.
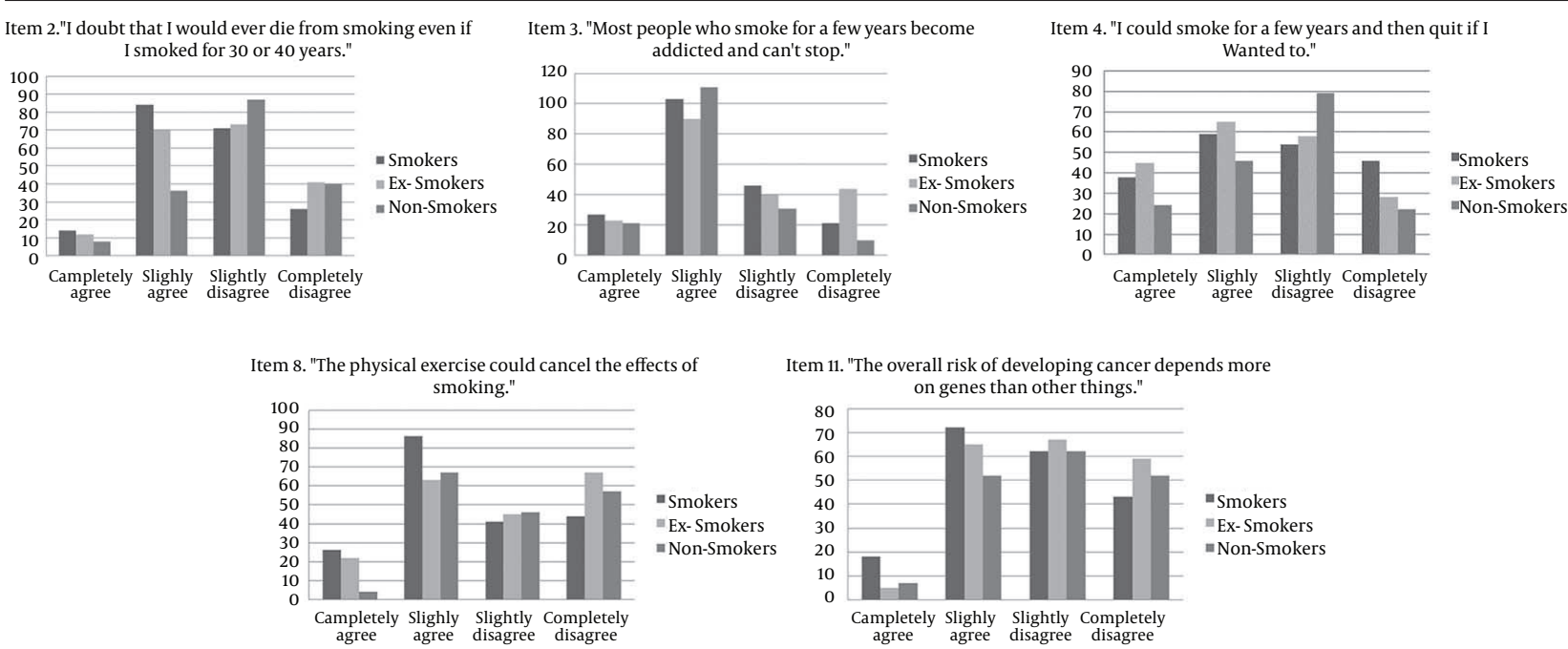

Figure 1. Distribution of Answers at Items 2, 3, 4, 8 and 11 
The aim of this study was to investigate the belief system in smokers and former smokers with respect to nonsmokers. The theoretical framework we used is based on the concept that the optimistic bias is structured on a set of mental models (cognitive architecture) used to appraise health-related risk in different contexts. Confirming our hypotheses, it emerged that the activation of a cognitive distortion is produced by an error in evaluating smoking-related risk. In particular, it was observed that smokers underestimate the strength of their dependence and related problems. Indeed, smokers generally consider having the control over their smoking behavior. In this way, smokers do not identify themselves as being abusers; however, at the opposite they believe that their behavior is the result of a hedonistic (the pleasure of tobacco) and aware evaluation $(5,14)$.

Our data have also confirmed a higher attitude of younger smokers to neglect the risk to develop a strong dependence due to nicotine absorption. Indeed, smokers between 19-40 years old consider the cigarette smoking as a consciously driven behavior. Only later, after repeated failed quit attempts, they come to understand their dependence to nicotine. Indeed, at the item 4 the answers of over 60 smokers showed that the awareness of their dependence is greater than in younger smokers. The obtained results from this study are in agreement with those of the previous studies (28-31), which have stressed both the experiential dimension of addiction and the limited capacity of people to rationally assess future consequences of their behavior (7) understanding the real complexity of a psychological and physical dependence to nicotine (5).

Another important result is the attitude of female smokers to underestimate smoking-related risks compared to males. This could explain the problem of the female smokers to give up smoking and the great risk of a new female smoking epidemic in some country, such as Italy (32). Previous research addressed this issue (33), finding associations between the fear to increase the body weight (aesthetics issues) and the perceived stress reported during the abstinence. This association might lead female smokers to underestimate risks to sustain smoking also in the presence of long-term negative consequences, preferring short-term benefits.

Actually, female smokers comparing with male smokers reported to believe that cigarette smoking has a preventive action against anxiety and depression and this belief is a serious obstacle to smoking discontinuation. Moreover, some researchers (34) have observed that females are more inclined to strain the disadvantages provoked by the smoking cessation, instead of the benefits. This is a salient factor, because the assessment of benefits and the identification of potential risks are related both to the give-up motivation and at the success rate of smoking cessation programs. Indeed, smokers with high level of motivation to give up are generally able to interrupt and to maintain the abstinence over a long time period (35).

Finally, the tendency to overestimate the efficacy of preventive strategies to contrast the negative effects of ciga- rettes (e.g. to carry out regular physical exercise, to adopt a healthy diet, to increase vitamins intakes and so on) was confirmed. Smokers tend to adopt a series of preventive behaviors with the belief to decrease risks for their health. Regarding this issue, it is interesting to compare the beliefs system of smokers and former smokers. We hypothesized that also former smokers need such beliefs in order to sustain their abstinence.

A high level of awareness about smoking-related risks might be considered the first motivational factor to contrast the pleasure associated with the cigarette consumption. Actually, they reported strong convictions about the hazardous effects of smoking. Furthermore, ex-smokers seem to have developed a considerable trust in the power of a healthy lifestyle to contrast all the risks associated with the previous smoking behaviors. In this way, former smokers may sustain their abstinence by believing that now they elude smoking-related risks thanks to healthier choices. Otherwise, thinking that the tobacco consumption had already impacted their health irremediably could weaken the decision to remain abstinence.

In conclusion, smokers and former smokers showed a different beliefs system. In particular, ex-smokers tend to assume the responsibility of their health, overestimate the impact of their decision, while smokers are more optimistic on their future and on their capacity to monitor health consequences. This optimistic perspective seems to put on the subject a "veil of Maya", which changes how the reality is seen, leading to harmful behaviors by overshadowing rational judgments. The unrealistic optimism is an important obstacle to interrupt the smokers' attempts, because it prevents the transition to the full awareness of tobacco consumption. This lack of awareness hinders the passage through the spiral of change (36) by developing the necessary motivation to quit.

Unfortunately, contrasting this optimistic distortion is not an easy task (1): however, developing of a strong individual awareness is fundamental to improve the likelihood of the adaptation of a healthy behavior. We argue that the comprehension of the cognitive processes of smokers is an important starting point, since it allows the understanding of the complex nature of smoking to promote tobacco cessation interventions able to fit with smoker needs. Since many antismoking interventions are only based on drugs, behavioral change and nicotine substitution strategies without considering the cognitive issues, investigate how smokers thinks about their behaviors and their risks is particular important. In particular, the use of the electronic cigarettes that promises to be the next frontier to contrast tobacco consumption in the near future should be analyzed within this framework to avoid the substitution of bad behavior with another one.

In conclusion, our data showed interesting suggestions to better understand the smoker's mind. However, this study has several limitations, in particular, the quantity and the quality of the sample and the data collection methodology, which do not allow drawing easy generalizations, since 
this is a survey study that do not permit variable manipulations. For this reason, we have limited the complexity of the data analysis to allow a simple and direct reading. However, we argue that our data suggest future lines of research able to verify the size effect and generalizability of our results other than to promote cognitive-based intervention to improve the adoption of healthy lifestyle and increase the efficacy of antismoking cessation programs.

\section{Authors' Contributions}

Dr. Marianna Masiero, Prof. Claudio Lucchiari and Prof. Gabriella Pravettoni designed the study and wrote the protocol. Dr. Marianna Masiero managed the literature searches and summaries of previous related works. Prof. Claudio Lucchiari undertook the statistical analysis, and Dr. Marianna Masiero wrote the first draft of the manuscript. All authors contributed to and have approved the final manuscript.

\section{Appendix}

Items Used to Evaluate Health Beliefs

\begin{tabular}{|l}
\hline Most People Who Smoke all Their Lives Eventually Die From an Illness \\
Caused by Smoking \\
\hline Completely agree \\
\hline Slightly agree \\
\hline Slightly disagree \\
\hline Completely disagree \\
\hline I doubt that I would ever die from smoking even if I smoked for 30 or 40 \\
\hline years \\
\hline Completely agree \\
\hline Slightly agree \\
\hline Slightly disagree \\
\hline Completely disagree \\
\hline Most people who smoke for a few years become addicted and can't stop. \\
\hline Completely agree \\
\hline Slightly agree \\
\hline Slightly disagree \\
\hline Completely disagree \\
\hline I could smoke for a few years and then quit if I wanted to \\
\hline Completely agree \\
\hline Slightly agree \\
\hline Slightly disagree \\
\hline Completely disagree \\
\hline How likely do you think that cigarette smokers will develop lung cancer? \\
\hline Very Low \\
\hline Moderate Discreet \\
\hline Very High \\
\hline How many people who have developed lung cancer do you think are cured? \\
\hline The $25 \%$ \\
\hline A $50 \%$ \\
\hline About $75 \%$ \\
\hline Almost all \\
\hline slightly higher lung cancer risk than \\
\hline double lung risk \\
\hline
\end{tabular}

The non-smokers lung cancer risk

Physical exercise could undo most of the effects of smoking.

Completely agree
Slightly agree
Slightly disagree
Completely disagree
Vitamins could undo most the effects of smoking.
Completely agree
Slightly agree
Slightly disagree
Completely disagree
There is no risk of getting cancer if you only smoke a few years.
Completely agree
Slightly agree
Slightly disagree
Completely disagree
The overall risk of getting cancer depends more on genes than anything
else
Completely agree
Slightly agree
Slightly disagree
Completely disagree

\section{References}

1. Weinstein ND, Klein WM. Resistance of personal risk perceptions to debiasing interventions. Health Psychol.1995;14(2):132-40.

2. Magnan RE, Koblitz AR, Zielke DJ, McCaul KD. The effects of warning smokers on perceived risk, worry, and motivation to quit. Ann Behav Med. 2009;37(1):46-57.

3. Balevich EC, Wein ND, Flory JD. Cigarette smoking and measures of impulsivity in a college sample. Subst Abus. 2013;34(3):256-62.

4. Viscusi WK. Smoking: Making the Risky Decision.Oxford: Oxford University Press; 1992.

5. Slovic P. Smoking. Risk, Perception \& Policy.California: Sage Publications; 2001.

6. Slovic P, Finucane ML, Peters E, MacGregor DG. The affect heuristic. Eur J Oper Res . 2007;177(3):1333-52.

7. Loewenstein GF, Weber EU, Hsee CK, Welch N. Risk as feelings. Psychol Bull. 2001;127(2):267-86.

8. Tyc VL, Hadley W, Allen D, Varnell S, Ey S, Rai SN, et al. Predictors of smoking intentions and smoking status among nonsmoking and smoking adolescents. Addict Behav. 2004;29(6):1143-7.

9. McKee SA, O'Malley SS, Salovey P, Krishnan-Sarin S, Mazure CM. Perceived risks and benefits of smoking cessation: gender-specific predictors of motivation and treatment outcome. Addict Behav. 2005;30(3):423-35.

10. Schnoll RA, Rothman RL, Newman H, Lerman C, Miller SM, Movsas B, et al. Characteristics of cancer patients entering a smoking cessation program and correlates of quit motivation: implications for the development of tobacco control programs for cancer patients. Psychooncology. 2004;13(5):346-58.

11. Hay JL, Ostroff J, Burkhalter J, Li Y, Quiles Z, Moadel A. Changes in cancer-related risk perception and smoking across time in newly-diagnosed cancer patients. J Behav Med. 2007;30(2):131-42.

12. Arnett JJ. Optimistic bias in adolescent and adult smokers and nonsmokers. Addict Behav. 2000;25(4):625-32.

13. Branstrom R, Kristjansson S, Ullen H. Risk perception, optimistic bias, and readiness to change sun related behaviour. Eur J Public Health. 2006;16(5):492-7.

14. Weinstein ND, Marcus SE, Moser RP. Smokers' unrealistic optimism about their risk. Tob Control. 2005;14(1):55-9.

15. Weinstein ND, Klotz ML, Sandman PM. Optimistic biases in public perceptions of the risk from radon. Am J Public Health 1988;78(7):796-800.

16. Klein CTF, Helweg-Larsen M. Perceived Control and the Optimistic Bias: A Meta-Analytic Review. Psychol Health. 2002;17(4):437- 
17. Ruthig JC, Haynes TL, Perry RP, Chipperfield JG. Academic optimistic bias: Implications for college student performance and well-being. Soc Psychol Educ. 2007;10(1):115-37.

18. Brown SL, Morley AM. Risk perception, fuzzy representations and comparative optimism. BrJPsychol. 2007;98(Pt 4):575-87.

19. Luo J, Isaacowitz DM. How optimists face skin cancer information: Risk assessment, attention, memory, and behavior. Psychol Health. 2007;22(8):963-84.

20. Petri H, Maldonato D, Robinson NJ. Data-driven identification of co-morbidities associated with rheumatoid arthritis in a large US health plan claims database. BMC Musculoskelet Disord. 2010;11:247.

21. Hoorens V, Buunk BP. Social Comparison of Health Risks: Locus of Control, the Person-Positivity Bias, and Unrealistic Optimism1.J Appl Soc Psychol. 1993;23(4):291-302.

22. Otten W, Van Der Pligt J. Context effects in the measurement of comparative optimism in probability judgments. J Soc Clin Psychol.1996;15(1):80-101.

23. Weinstein ND. Unrealistic optimism about future life events. $J$ Pers Soc Psychol. 1980;39(5):806-20.

24. Weinstein ND. Why it won't happen to me: perceptions of risk factors and susceptibility. Health Psychol. 1984;3(5):431-57.

25. Leventhal H, Glynn K, Fleming R. Is the smoking decision an 'informed choice'? Effect of smoking risk factors on smoking beliefs. JAMA. 1987;257(24):3373-6.

26. Heatherton TF, Kozlowski LT, Frecker RC, Fagerstrom KO. The Fagerstrom Test for Nicotine Dependence: a revision of the Fagerstrom Tolerance Questionnaire. BrJ Addict. 1991;86(9):1119-27.
27. Marino LL. disassuefazione dal fumo: L'ambulatorio. In: Nardini S, Donner C editors. L'epidemia di fumo in Italia.. Pisa: EDI-AIPO Scientifica; 2000 .

28. Borrelli B, Hayes RB, Dunsiger S, Fava JL. Risk perception and smoking behavior in medically ill smokers: a prospective study. Addiction. 2010;105(6):1100-8.

29. Oncken C, McKee S, Krishnan-Sarin S, O'Malley S, Mazure CM. Knowledge and perceived risk of smoking-related conditions: a survey of cigarette smokers. Prev Med. 2005;40(6):779-84.

30. Ayanian JZ, Cleary PD. Perceived risks of heart disease and cancer among cigarette smokers. JAMA. 1999;281(11):1019-21.

31. Strecher VJ, Kreuter MW, Kobrin SC. Do cigarette smokers have unrealistic perceptions of their heart attack, cancer, and stroke risks? J Behav Med. 1995;18(1):45-54.

32. Fumo.. Rapporto sul fumo in Italia 2013.: Istituto Superiore di Sanità;. Available from: http://www.iss.it/fumo/index. php?lang=1\&id=318\&tipo=18.

33. Toll BA, Salovey P, O'Malley SS, Mazure CM, Latimer A, McKee SA. Message framing for smoking cessation: the interaction of risk perceptions and gender. Nicotine Tob Res. 2008;10(1):195-200.

34. Bock BC, Fava JL, Gaskins R, Morrow KM, Williams DM, Jennings E, et al. Yoga as a complementary treatment for smoking cessation in women. J Womens Health (LarChmt). 2012;21(2):240-8.

35. Perkins KA. Smoking cessation in women. Special considerations. CNS Drugs. 2001;15(5):391-411.

36. DiClemente CC, Prochaska JO, Fairhurst SK, Velicer WF, Velasquez MM, Rossi JS. The process of smoking cessation: an analysis of precontemplation, contemplation, and preparation stages of change.J Consult Clin Psychol. 1991;59(2):295-304. 